\title{
Implantologie et épithèses faciales
}

\section{RÉSUMÉ}

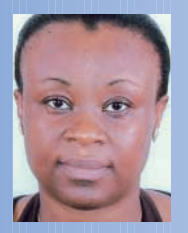

Yomin Cécile ALLOH AMICHIA

Docteur en chirurgie dentaire,

Maître-assistant

à la faculté de chirurgie dentaire d'Abidjan, Assistante associée des Universités

à la faculté de chirurgie dentaire de Nantes, Département de prothèses,

1, place Alexis Ricordeau,

BP 84215,

44042 Nantes Cedex 01.

François BRÉTÉCHÉ

Docteur en médecine,

Stomatologue,

68, boulevard Meusnier-de-Querlon,

44200 Nantes.

\section{Joël LANHOUET}

Épithésiste-oculariste,

28, rue Jacques-Prévert,

44200 Nantes.

\section{Laurent LE GUEHENNEC}

Docteur en chirurgie dentaire,

MCU-PH à la faculté de chirurgie dentaire de Nantes,

Département de prothèses,

1, place Alexis Ricordeau,

BP 84215 ,

44042 Nantes Cedex 01.

\section{Pierre LE BARS}

Docteur en chirurgie dentaire,

MCU-PH à la faculté de chirurgie dentaire

de Nantes,

Département de prothèses,

1, place Alexis Ricordeau,

BP 84215 ,

44042 Nantes Cedex 01.
Les épithèses faciales conservent leurs indications aujourd'hui encore dans certaines situations cliniques. Les fixations classiques telles que les adhésifs, les colles, les lunettes, sont remplacées par I'utilisation d'implants extra-oraux en titane à ancrage osseux. Ceux-ci constituent une alternative fiable et sécurisante en prothèse maxillo-faciale, en offrant aux patients une meilleure rétention de l'épithèse. Les prothèses peuvent alors être positionnées par des fixations soit mécaniques, soit magnétiques, soit mixtes en associant les deux. 


\section{Introduction}

$>$

Les prémices des prothèses faciales remontent à l'Antiquité. Des masques faciaux de 2500 ans avant notre ère ont été trouvés lors de fouilles archéologiques et avaient pour objectif de restaurer des visages. Avec Ambroise Paré, la prothèse maxillo-faciale prend un nouvel envol au $x \mathrm{xl}^{\mathrm{e}}$ siècle par la réalisation de prothèses. Au cours de I'histoire, de nombreux matériaux tant rigides (l'or, l'argent plus lourds) que souples (le latex, la vulcanite) ont été utilisés pour la fabrication des épithèses [1, 2]. Aujourd'hui, la prothèse plastique ou épithèse faciale consiste en un dispositif principalement esthétique, ayant pour vocation de combler et de dissimuler les pertes de substance congénitales ou acquises (traumatiques ou chirurgicales) de tout ou partie du visage humain $[2,3]$. Ces défauts exobuccaux [4] de diverses origines sont préjudiciables et portent atteinte à l'identité du patient. La réalisation d'une épithèse a donc un rôle à la fois cosmétique et fonctionnel, mais permet aussi de contribuer au bien-être moral et physique du patient d'où son rôle psychothérapeutique. On distingue plusieurs sortes d'épithèses: de la pyramide nasale, les artifices oculo-palpébraux, du pavillon de l'oreille, les reconstructions hémifaciales ou les larges défects maxillo-faciaux [5].

La prothèse faciale vient compléter le travail chirurgical quand celui-ci a atteint ses limites esthétiques et réparatrices (rejet de greffes, impossibilités de reconstructions dans le cas d'exérèse large, os fragilisé par la radiothérapie...). Elle peut être aussi réalisée en attente de la chirurgie reconstructive. Cette réhabilitation prothétique présente l'avantage de reconstituer fidèlement les organes absents ou défectueux grâce à la technique du moulage facial. Elle est aussi réalisée en ambulatoire et propose une réponse efficace au problème psychologique du malade. Son caractère amovible rend possible la surveillance des tissus sous-jacents et sa mise en œuvre permet d'attendre ou de compenser la chirurgie réparatrice. La prothèse faciale reste donc d'actualité $[6,7]$.

La réalisation de la prothèse faciale externe peut se heurter à des problèmes de rétention qui paraissent insolubles malgré tout le soin apporté à leur réalisation [8]. Plusieurs moyens de rétention sont à disposition. Leur utilisation ne relève pas de choix mais d'indications précises en fonction du cas clinique et de la région anatomique à réhabiliter $[9,10]$ :

- la rétention mécanique exploite les contredépouilles, des cavités naturelles ou artificielles conséquences de la chirurgie d'exérèse ;

- les montures de lunettes indiquées pour les épithèses provisoires rendent de grands services dans une large majorité de cas. Elles constituent un élément de stabilisation de premier ordre. Il est important d'obtenir une amovibilité entre la monture et l'épithèse (pour un nez) afin d'en faciliter l'entretien ;

- les adhésifs utilisés dans certaines conditions vont permettre une stabilisation de la prothèse et augmentent de ce fait le confort du malade.

Malgré la mise en œuvre de tous ces moyens, la rétention de l'épithèse n'est pas toujours parfaitement obtenue. On observe des réactions cutanées avec des prothèses adhésives, des problèmes de rétention et de camouflage des bords $[12,13]$. Aussi, le recours à des implants permanents transcutanés en titane 
va-t-il permettre d'améliorer grandement ces problèmes de fixation [11]. Depuis leur première utilisation à la fin des années 1970 , ils ont pour but d'augmenter la qualité de la réhabilitation mais aussi d'éviter certaines reconstructions chirurgicales lourdes n'atteignant pas toujours les qualités fonctionnelles et esthétiques escomptées. En plus des fixtures endo-osseuses issues des travaux de Bränemark, le développement des implants juxta-osseux élargit les indications pour les épithèses. Leur utilisation permet d'éviter ou du moins de réduire au minimum les inconvé- nients des autres types de fixation. Ils permettent deux types de fixation: mécanique et magnétique. Cette dernière est surtout réservée au cadre orbitaire où ne se posent pas de problèmes de contraintes mécaniques. La fixation est mécanique au pavillon de l'oreille et mixte dans les défects faciaux.

Cet article passe en revue, à partir de quelques cas cliniques, les contre-indications et les indications des implants extra-oraux, et la mise en œuvre des principales épithèses concernant la face, avec leurs avantages et leurs inconvénients.

\section{Indications et sites implantaires : applications cliniques}

\section{Indications}

L'indication première des épithèses implantoportées concerne tous les cas où la chirurgie reconstructrice ne peut intervenir de façon satisfaisante, c'est-à-dire concerne les cas de difficulté majeure de reconstruction chirurgicale rendant celle-ci aléatoire et peu satisfaisante sur le plan esthétique et morphologique, les échecs de reconstruction chirurgicale du fait des cicatrices ou du souhait du patient [15].

Ces épithèses peuvent aussi servir de solution d'attente, que ce soit en traumatologie (surtout balistique) pour permettre une intégration sociale et familiale correcte ou en carcinologie afin de s'assurer de la non-évolutivité de la tumeur à court et moyen terme avant d'envisager une reconstruction autologue.

Les épithèses sont aussi indiquées chez les patients présentant un état général précaire contre-indiquant une chirurgie lourde ou en plusieurs temps ou lorsque le site nécessite une surveillance locale ou loco-régionale devant un pronostic incertain.

Enfin, le recours à une épithèse peut aussi être le souhait du patient qui, pour des raisons personnelles et/ou socioprofessionnelles, peut préférer un mode de réhabilitation rapide, simple, avec un résultat esthétique prévisible et à peu près satisfaisant obtenu plus rapidement $[15,18]$.

\section{Sites implantaires}

Jensen et al. [29] ont évalué sur 15 crânes secs anatomiques les volumes osseux disponibles identifiant ainsi les sites potentiels d'implantation. Concernant les fixtures extra-orales, trois principales aires d'implantation sont répertoriées : la région nasale et naso-maxillaire, la région auriculo-temporale et la région oculopalpébrale $[20,15]$. Sur ces implants, le mode de fixation recherché doit être d'abord mécanique (barre et clip de préférence), puis mixte 
et enfin magnétique en derniers recours $[5,21]$. La rétention mécanique par clip est utilisée pour les larges pertes de substances [22].

\section{> Région auriculo-temporale}

\section{- Indications}

Après de multiples opérations, il se forme un tissu cicatriciel et fibreux ne permettant pas la reconstruction plastique. La prothèse auriculaire offre alors l'avantage de minimiser le traumatisme esthétique. Dans les cas de brûlures étendues ou d'avulsions avec d'importantes dégradations des tissus locaux, la destruction du pédicule temporal superficiel n'autorise pas de constructions par lambeaux. Dans les cas d'amputations chirurgicales complètes du pavillon de l'oreille suite à une pathologie tumorale, le terrain n'est pas favorable pour la reconstruction [23]. Dans les malformations congénitales intéressant le pavillon de l'oreille et qui sont en général unilatérales, partielles ou totales, il peut exister des patho- logies associées (comme dans le syndrome de Franceschetti) rendant aléatoire la reconstruction chirurgicale. La prothèse auriculaire implanto-portée constitue alors une solution de choix en cas de contre-indication de la chirurgie esthétique (fig. 1 a à c).

Ces épithèses auriculaires sont réalisées dans des matières plastiques telles que les silicones qui offrent l'avantage d'être colorables et modelables, permettant des formes et des caractéristiques ressemblant fortement aux reliefs normaux. Associés à l'ancrage sur les implants, les artifices prothétiques peuvent être plus légers, plus souples et donc discrets. La prothèse est simplement clippée et sa durée de vie est aussi plus longue parce que les manipulations sont réduites.

\section{- Sites implantaires}

La région mastoïdienne semble être le site d'implantation favorable selon les études de Tjellström [24]. Il rapporte un taux de succès de $98 \%$ pour un suivi de plus de 350 patients. La
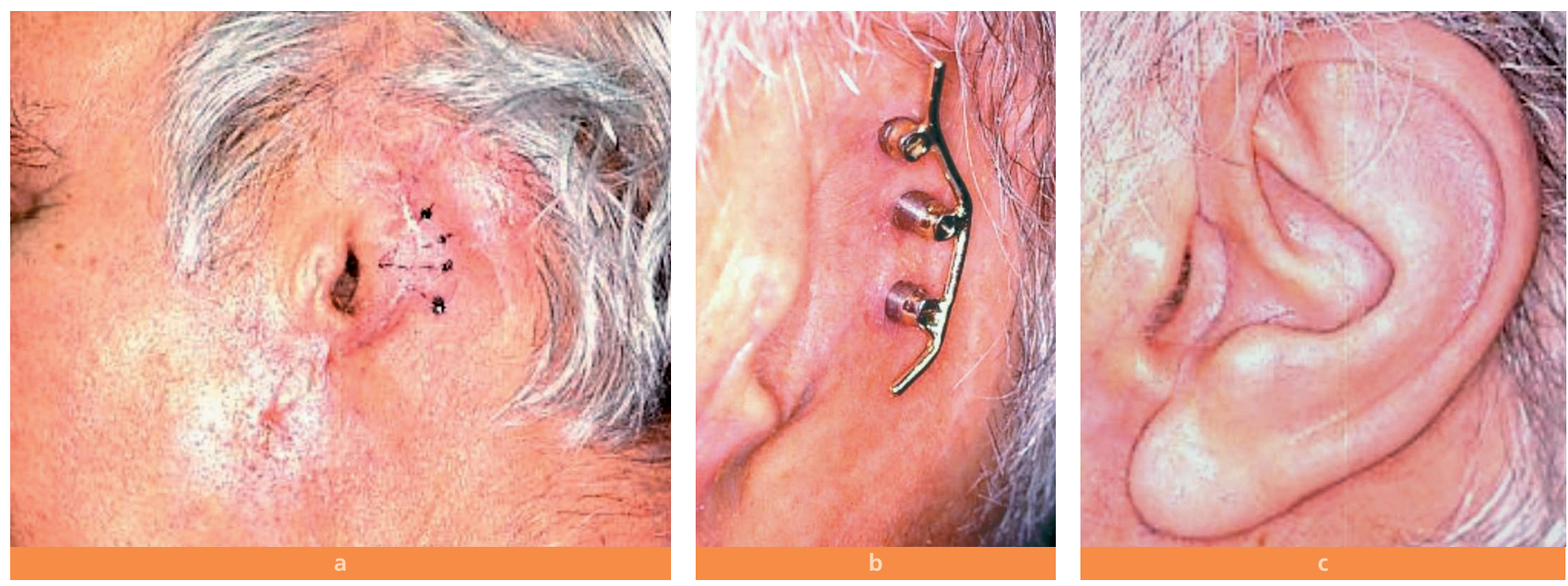

Fig. 1 a à c Épithèse du pavillon de l'oreille.

a : État initial : perte de substance auriculaire accidentelle ;

b : Implants transcutanés et barre de fixation de la prothèse ;

c : Épithèse en place. 
densité osseuse corticale de l'os temporal et la faible épaisseur des parties molles en regard de cette région sont deux facteurs qui expliquent ces résultats. Pour Telljström, la localisation optimale du site implantaire se situe sur un arc de cercle à $18 \mathrm{~mm}$ en arrière du centre du conduit auditif externe [24]. Pour la plupart des auteurs $[20,25,26,27]$, deux implants extraoraux ostéo-intégrés sont suffisants, les dimensions tolérables au niveau de l'os temporal de 3 à $4 \mathrm{~mm}$ permettant un ancrage suffisant. Un guide chirurgical est nécessaire pour déterminer la meilleure position de l'implant [20, 26].

À ces fixtures, divers types d'attachement peuvent être associés : les attachements type clips sont les plus utilisés. La fixation magnétique est très peu utilisée car insuffisante : le poids des lunettes tend en effet à déplacer la prothèse $[19,27]$.

\section{> Région nasale}

\section{- Indications}

La reconstruction chirurgicale complète de la pyramide nasale représente une difficulté chirurgicale importante en raison de la nécessité de modeler trois plans: muqueux, ostéocartilagineux et cutané [23]. Aussi cette intervention n'est-elle envisagée qu'en présence de perte de substance partielle, avec des structures cartilagineuses suffisantes pour recevoir un lambeau [28]. Plusieurs temps opératoires sont nécessaires pour un résultat globalement modeste sur le plan morphologique. Cette réhabilitation chirurgicale de la pyramide nasale est de plus contre-indiquée chez le patient âgé, s'il est impossible d'obtenir un site donneur adéquat ou d'effectuer une surveillance du site. Seule la solution épiprothétique reste alors possible.

Autrefois, les prothèses nasales étaient retenues par des adhésifs ou associées à des mon- tures de lunettes. L'évacuation des sécrétions nasales, la condensation issue de la respiration et du tabac ou les mimiques du visage pouvaient alors compromettre la stabilité prothétique [28]. Le recours à une épithèse implantoportée permet de s'affranchir des aléas de la rétention adhésive ou des ancrages sur montures de lunettes. Enfin, le recours à une épiprothèse permet de restituer quasiment ad integrum l'aspect initial du nez du patient et donc la physionomie du visage.

\section{- Sites implantaires}

Le site implantaire le plus favorable demeure au niveau du plancher antérieur des fosses nasales du fait de la densité osseuse $(10,2 \mathrm{~mm})$ [29]. Le site glabellaire de l'os frontal présente aussi une bonne densité osseuse mais reste peu vascularisé. Pour garantir la stabilité de la prothèse, trois implants sont nécessaires: deux au niveau du plancher antérieur des fosses nasales et un dans l'os frontal au niveau de la glabelle (fig. 2 a à c, fig. $\mathbf{3}$ a et b). Ces implants extra-oraux ou dentaires positionnés dans le plancher antérieur des fosses nasales doivent autoriser la sortie des piliers à l'intérieur des limites de l'épithèse nasale selon un angle d'environ $60^{\circ}$ par rapport à un plan horizontal [20].

L'orifice piriforme n'offre pas un ancrage suffisamment solide aux implants-vis extra-oraux : cette région est en effet plus propice à la résorption qu'à l'apposition osseuse. L'emploi d'implant-plaque permet de positionner les piliers au-dessus du vide que représentent les fosses nasales [20]. Les trois implants autorisent soit le maintien d'une barre en «T» inversée sur laquelle trois clips permettent l'immobilisation de la prothèse, soit des plateaux métalliques fixés sur des piliers et aimants incorporés à la face profonde de l'épithèse. Ces derniers sont sujets à la corrosion entraînant une discoloration de la prothèse [22]. 

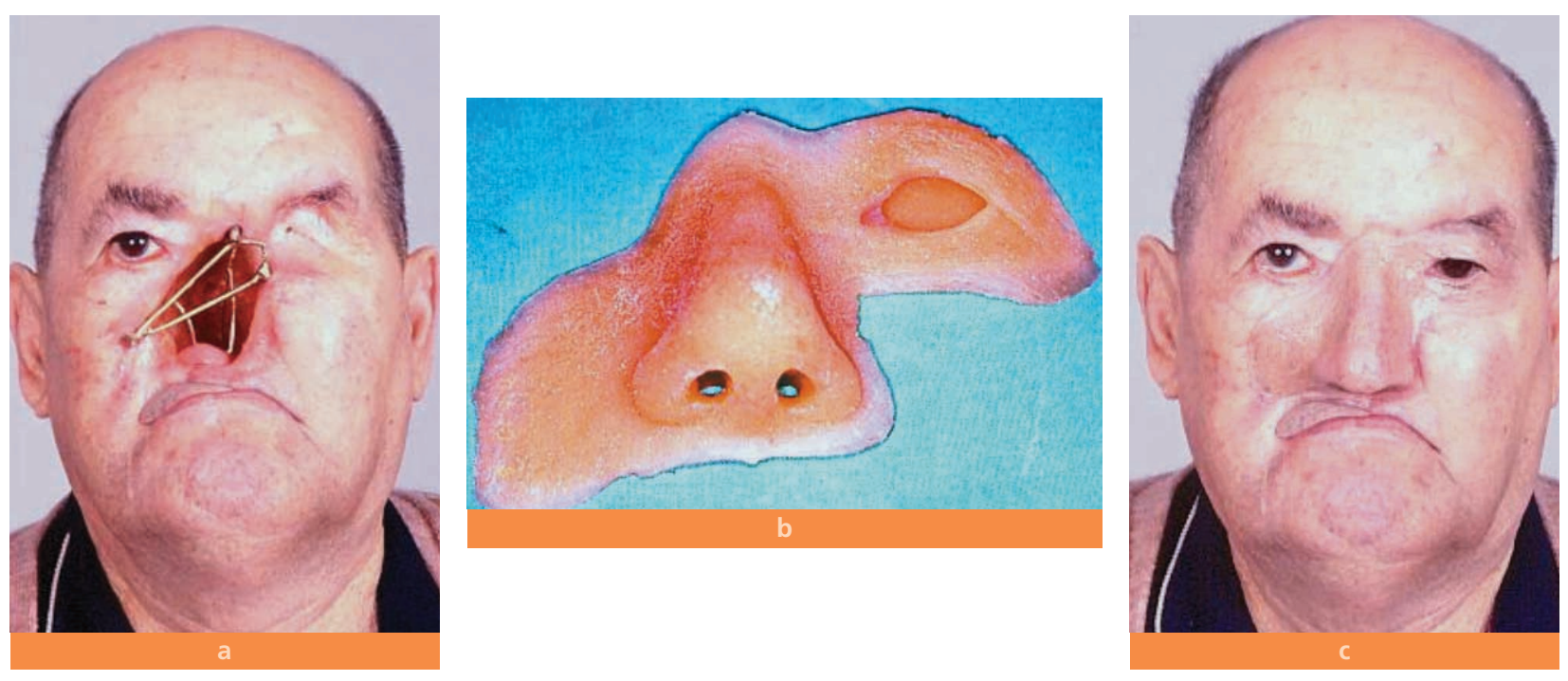

Fig. 2 a à c Épithèse nasale et orbitaire.

a : mise en place de la barre d'attachement sur les piliers.

b : épithèse.

c: épithèse en place.

Implantologie Dr François Brétéché.

Épithèses M. Joël Lanhouet.
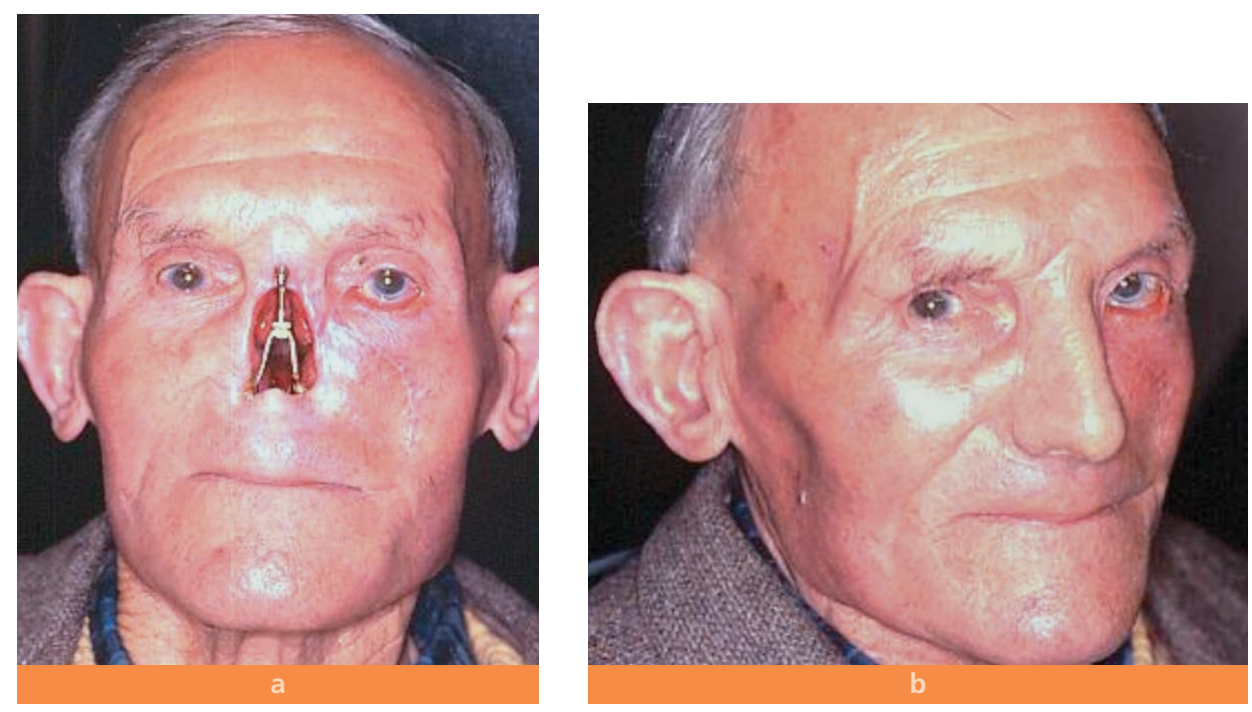

Fig. 3 a et $b$ Épithèse nasale.

a : implants avec la barre d'attachement.

b : épithèse nasale en place.

Implantologie Dr François Brétéché.

Épithèses M. Joël Lanhouet. 


\section{> Région oculo-palpébrale}

\section{- Indications}

La réhabilitation chirurgicale de ces pertes de substances nécessite de rétablir trois plans: osseux, muqueux et cutanés. Elle impose un certain nombre d'interventions. De préférence, le terrain osseux et cutané doit être sain, mais la plupart du temps le patient a bénéficié d'une irradiation [23]. La chirurgie plastique et reconstructrice atteint alors ses limites qualitatives et ne permet pas dès lors de restituer l'intégrité esthétique et morphologique de cette zone. Le recours aux épithèses implanto-portées constitue donc une alternative réparatrice intéressante. Ces prothèses faciales sont en résines méthyl-méthacriliques pour le globe oculaire (fig. $\mathbf{2}$ b et c) et en silicone pour les parties remplaçant la peau.

\section{- Sites implantaires}

Les sites propices à recevoir des implants au niveau du cadre orbitaire ont été déterminés par Jensen [29]. Le rebord infra-orbitaire (en dehors du nerf infra-orbitaire) et la colonne externe de l'orbite autorisent la mise en place d'implants extra-oraux de 3 à $7 \mathrm{~mm}$ de longueur et de $3,75 \mathrm{~mm}$ de diamètre, sous réserve d'une évaluation radiologique précise de l'épaisseur de l'os pour éviter une éventuelle effraction des sinus maxillaires. L'épaisseur osseuse moyenne dans la partie supéro-interne et supéro-externe au niveau du rebord supra-orbitaire est respectivement de 4,4 $\mathrm{mm}$ et de $4,6 \mathrm{~mm}$. En règle générale, le sinus frontal $n$ 'atteint pas la portion supéroexterne, ce qui en fait une zone d'implantation intéressante. Par ailleurs, la distance antéro-postérieure entre le rebord orbitaire supérieur et la partie la plus antérieure du lobe frontal varie de $6,3 \mathrm{~mm}$ à $14,5 \mathrm{~mm}$, ce qui implique de positionner les implants d'une façon quasi verticale vers la fosse crânienne afin d'éviter tout risque de brèche ostéoméningée [20]. Ces lits implantaires permettent une assez bonne ostéo-intégration même si une radiothérapie antérieure a été effectuée.

L'émergence cutanée des piliers solidarisés aux fixtures détermine le positionnement précis des implants. Les piliers doivent se situer en dedans des limites de la perte de substance, d'où l'importance du guide chirurgical permettant aussi d'apprécier les contours de la future épithèse. L'absence de contraintes mécaniques exercées sur la prothèse oculo-palpébrale permet d'utiliser seulement trois voire quatre implants pour la fixation de l'épithèse sur des plateaux métalliques vissés sur des piliers de $4 \mathrm{~mm}$ [20]. Les implants sont positionnés de telle sorte que leur prolongement puisse se réunir vers le milieu de l'orbite comme au sommet d'une pyramide afin d'obtenir une bonne stabilité. Ainsi chaque plateau magnétique $s^{\prime}$ inscrit dans la mesure du possible dans un des plans du trièdre pour éviter tout glissement latéral des aimants sur ces plateaux [23]. Pour des prothèses plus lourdes, l'épithésiste préfère utiliser des fixations magnétiques avec une barre et des cavaliers [23]. Le port des lunettes permet en plus de camoufler une partie de l'épithèse et d'assurer un ancrage supplémentaire dans les situations les plus difficiles. 


\section{Contre-indications}

Les contre-indications relatives ou absolues sont liées à des affections pour lesquelles l'acte chirurgical est à risque ou interfère avec la cicatrisation tissulaire [14]. Elles sont de trois ordres: générales, loco-régionales et locales. Selon Labbé et al. [15], concernant les implants extra-oraux, les contre-indications absolues sont exceptionnelles et relèvent du bon sens: ostéite, leucémie, lymphome, cirrhose à un stade terminal. Les contre-indications relatives relèvent également $d u$ bon sens: malade en phase terminale, absence totale d'hygiène, refus psychologique. Les contre-indications à l'anesthésie générale ne constituent pas, a priori, un obstacle puisque la pose des implants et des piliers peut être réalisée sous anesthésie locale. Les manipulations sont simples, stables et commodes. La frontière entre contre-indication relative et contre-indication absolue n'est pas formelle. Elle doit être adaptée à différents paramètres comme l'expérience du chirurgien. Aussi, pour Berdugo [16], les contre-indications sont actuellement quasi inexistantes. Les contreindications médicales sont la plupart du temps temporaires et, une fois la pathologie traitée, les traitements redeviennent possibles. Persiste toutefois le problème de leur insertion en territoire irradié. La dose, la durée d'irradiation et le délai écoulé depuis la fin de la radiothérapie sont des paramètres à évaluer avant la pose des fixtures $[2,17,18]$.

\section{Classification, choix,} protocole chirurgical de mise en place des implants extra-oraux et temps prothétique

\section{Classification}

La fixation de l'épithèse implanto-portée, à la différence de la prothèse maxillo-faciale classique, est assurée par des piliers transcutanés. Le pilier est solidarisé à une pièce métallique fixée à l'os sous-jacent et appelé implant extraoral dont il existe deux grandes catégories: les implants endo- et juxta-osseux (fig. 4 a à c). Les implants endo-osseux découlent des implants initialement développés par
Bränemark et sont commercialisés par de nombreuses sociétés (Nobelpharma, Straumann, Bud Industries, IMZ) [30]. Pour ce type d'implant, une épaisseur d'os minimum de $3 \mathrm{~mm}$ reste nécessaire. La deuxième catégorie, les implants juxta-osseux, ne nécessite pas cette épaisseur minimale (fig. 4 d). Ce sont le système Epitec de Leibinger et l'implant-plaque pour os plat (IPOP). La différence entre un implant IPOP et l'implant-plaque consiste en une simplification de préparation de la plaque $[2,30,31]$. 

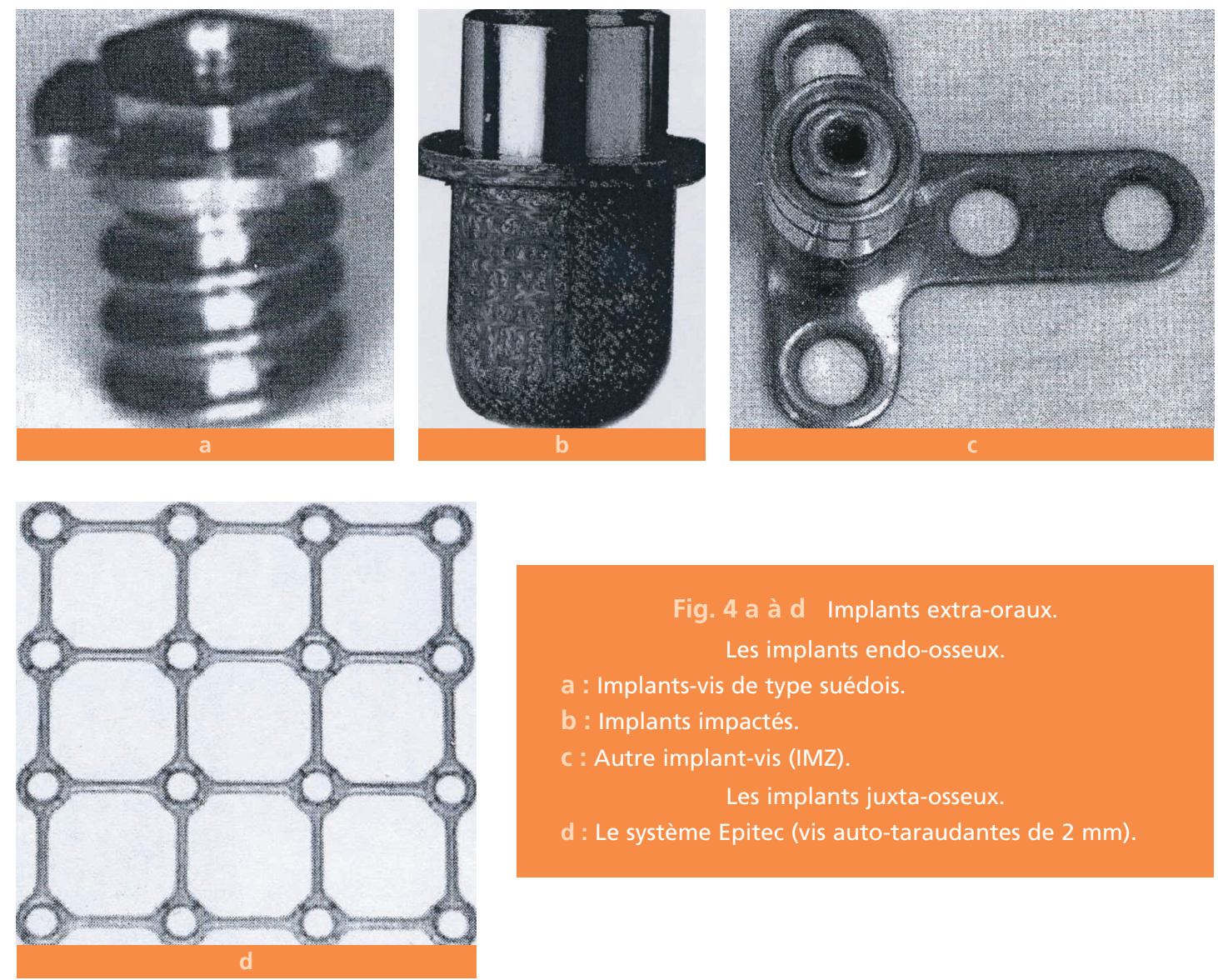

Fig. 4 à $\mathrm{d}$ Implants extra-oraux.

Les implants endo-osseux.

a : Implants-vis de type suédois.

b : Implants impactés.

c: Autre implant-vis (IMZ).

Les implants juxta-osseux.

d: Le système Epitec (vis auto-taraudantes de 2 mm).

\section{Choix}

La structure de certains os de la face comme le frontal ou l'os zyggomatique se prête à l'usage des implants-vis. Ces implants sont indiqués dans les épithèses orbito-palpébrales. Lorsque le support osseux est insuffisant comme au niveau du pourtour de l'orifice piriforme ou de la région mastoïdienne, le système Epitec représente une alternative intéressante [2, 32]. L'indication de l'implant-plaque reste la réhabilitation nasale étendue, mais aussi parfois la prothèse de pavillon d'oreille en cas de cellules mastoïdiennes nombreuses et volumineuses ne permettant pas I'utilisation des implantsvis [33]. Pour ces applications extra-orales, les implants utilisés sont en titane de 3 à $4 \mathrm{~mm}$ de longueur et d'un diamètre de 3,5 mm [2]. Un rebord sur la fixture empêche la pénétration excessive dans l'os du crâne lors de la mise en place [34].

Quel que soit le type d'implant utilisé, la détermination des sites implantaires nécessite une phase préopératoire. Elle comprend des clichés qui varient selon le type de perte de substance : crâne, face, profil, téléradiographies, cadre orbitaire. Pour une appréciation précise de l'épaisseur du support osseux et d'éventuels obstacles anatomiques (sinus, cellules ethmoïdes, gros vaisseaux), des coupes scanner sont nécessaires. Ces clichés aident à l'appréciation des tissus mous en regard des sites d'implantation prévus et donc au choix du type d'implant [30, 32]. Pendant cette phase, l'apport de l'épiprothésiste reste indispensable 
pour la réalisation du guide chirurgical en résine, silhouette de la future prothèse et sur lequel sont matérialisées les émergences souhaitables des piliers transcutanés $[2,30,32,35]$. Le choix du type, du nombre et de la position des implants se fait en étroite collaboration avec l'épithésiste et aboutit à la réalisation d'une maquette de simulation.

\section{Protocole chirurgical}

Deux temps opératoires sont généralement nécessaires (technique des implants enfouis). Le premier temps chirurgical débute par l'incision cutanée, la réduction éventuelle des tissus mous sous-cutanés et le décollement périosté. Le forage suivi éventuellement d'un taraudage permet la mise en place des implants recouverts de la vis de couverture. Des sutures hermétiques ferment le site opératoire. Le second temps chirurgical consiste en la découverte de l'implant et la pose du pilier, trois à huit mois plus tard. Ce délai de mise en nourrice dépend du terrain osseux (irradié ou non), de la localisation des implants (os très corticalisé ou non) et de l'indication (prothèse auditive à ancrage endo-osseux ou prothèse maxillofaciale) [30]. Après incision et ablation de la vis de couverture, le pilier est positionné et coiffé par un capuchon de cicatrisation $[2,33]$ (fig. 5 b à d), avant la suture de la zone périimplantaire. Certains auteurs proposent un protocole de pose de l'implant et du pilier en un seul temps [33]. Le pilier percutané est laissé hors charge pendant deux mois avant sa mise en fonction.

Pour les implants-plaques, le protocole de mise en place est proche de celui d'une ostéosynthèse. Comme pour les implants endo-osseux, la procédure comporte deux temps chirurgicaux, parfois réalisés en un temps. Dans un premier temps, l'abord cutané et périosté est réalisé plus largement que pour les implantsvis. La plaque, adaptée au relief, est ostéosyn-
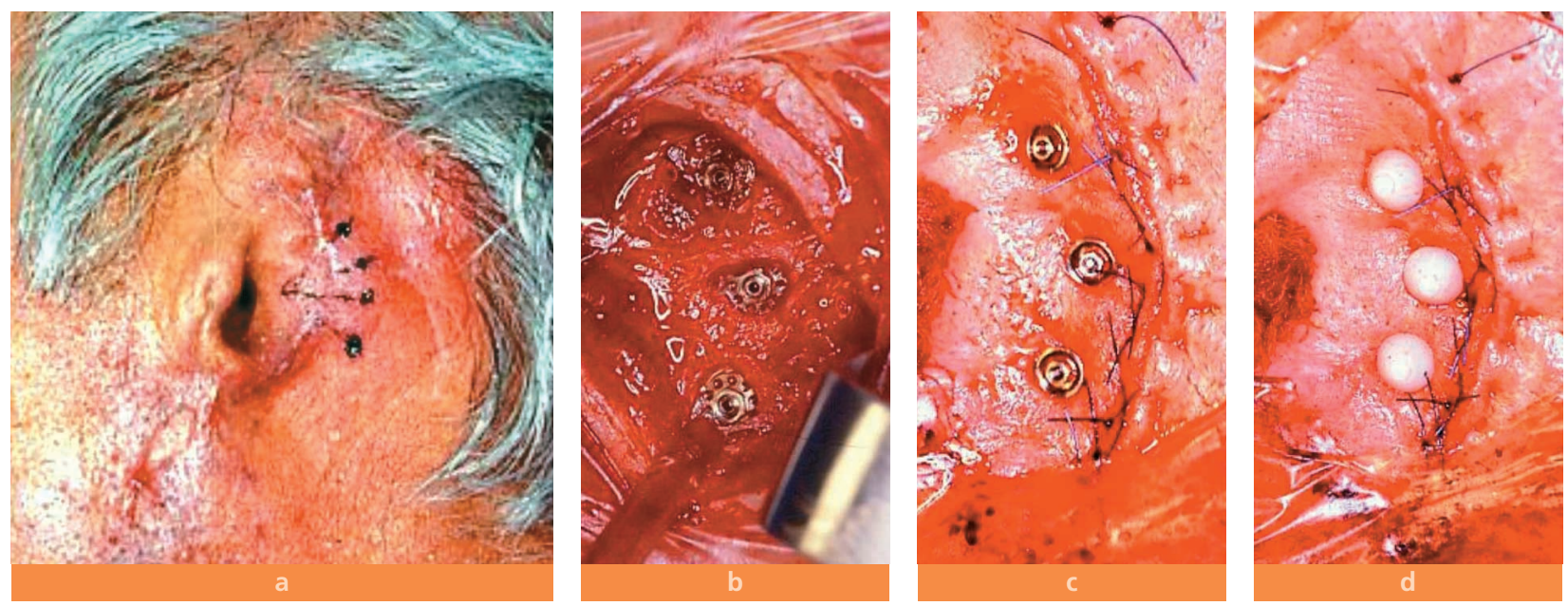

Fig. 5 a à d Épithèse auriculaire.

a : localisation des sites implantaires à l'écaille du temporal.

b : pose chirurgicale des implants de 3 à $4 \mathrm{~mm}$ de diamètre et piliers endo-osseux.

c : amincissement de la peau et suture.

d : mise en nourrice des implants. 
thésée par des vis, les orifices destinés aux piliers étant obturés par des vis capuchons d'attente. Une réduction des tissus mous souscutanés termine l'intervention avant les sutures. Dans le deuxième temps, les vis d'attente sont remplacées par les piliers percutanés recouverts par des capuchons de protection. La suture se réalise à la suite. Elle est séparée pour les plans périosté et cutané et identique pour les implants-vis ou les implantsplaques [2].

\section{Temps prothétique}

Les temps prothétiques sont comparables à ceux de la prothèse implanto-portée. En préopératoire, la prise d'empreinte aux alginates de la perte de substance permet la confection du guide chirurgical et la sélection sur celui-ci des points d'émergence cutanés des piliers. En post-chirurgical, le temps prothétique débute après cicatrisation cutanée complète, soit 15 jours à 3 semaines après le second temps
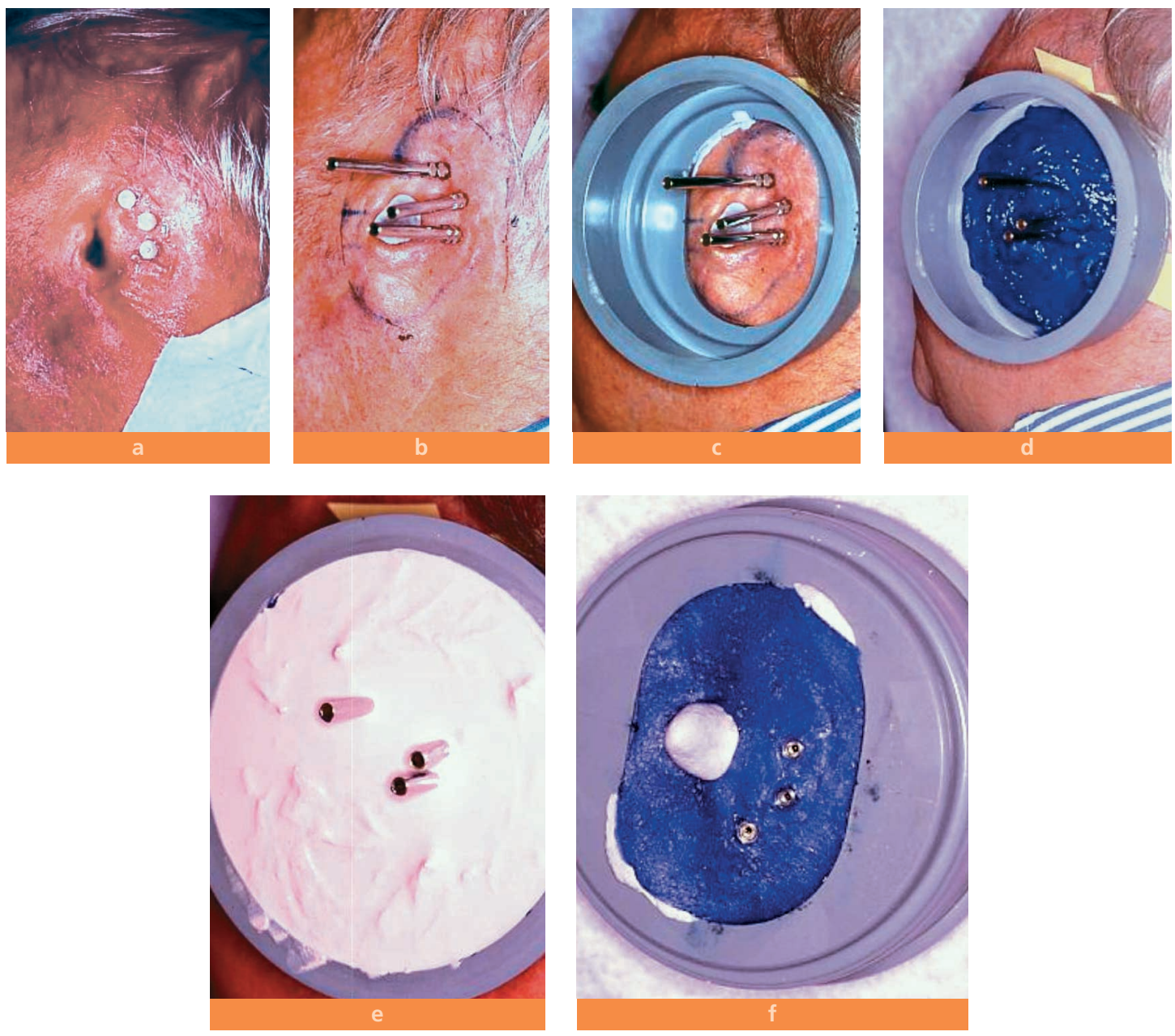

Fig. 6 a à f Les différentes étapes de la prise d'empreinte en vue de la réalisation d'un pavillon de l'oreille.

a : implants en nourrice.

b : mise en place des transferts avant la prise d'empreinte.

c: coffrage avant l'empreinte. d: empreinte à l'aide d'un silicone de type Xantopren.

e : sur-empreinte au plâtre.

f : intrados de l'empreinte. 

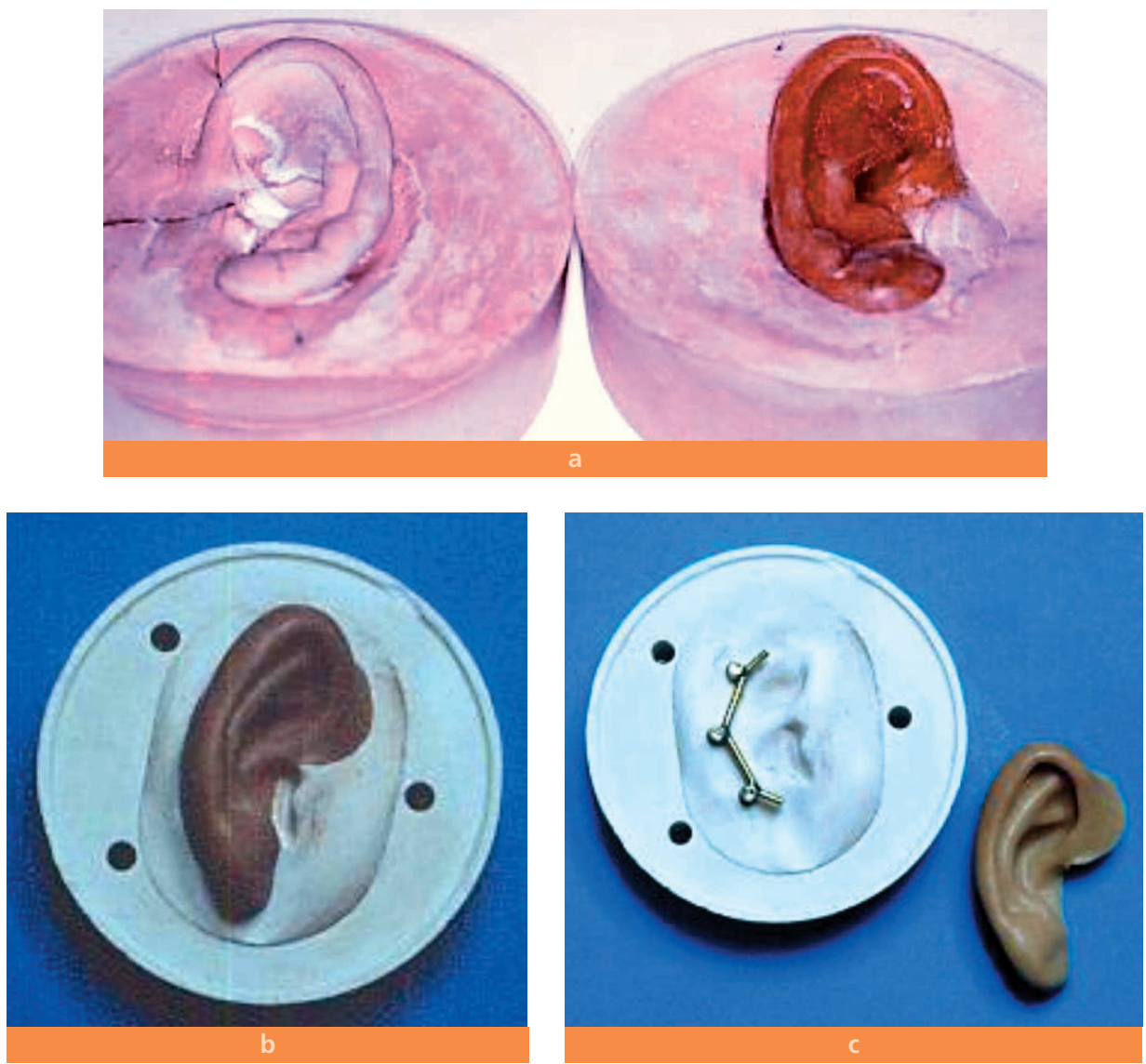

Fig. 7 a à c Reproduction de l'oreille selon I'homothétique

a : moulage en plâtre de l'oreille saine et reproduction en cire de l'épithèse selon I'homothétique

b : pavillon de l'oreille reconstruite en silicone

$c$ : barre d'attachement de l'épithèse.

chirurgical et en accord avec le chirurgien [33]. Avec un porte-empreinte individuel, l'enregistrement des piliers transcutanés peut alors être réalisé afin de confectionner un modèle de réplique de la position des piliers. Ensuite est confectionnée la plaque base qui diffère selon le type de fixation, magnétique ou mécanique.
La cire de l'épithèse se monte sur cette plaquebase. La validation de la maquette en cire se fait après essayage, et avant la réalisation d'un moule de cuisson. La cuisson et le moulage de l'épithèse sont alors effectués [2] (fig. 6 a à f, fig. 7 a à c). 


\section{Comparaison}

\section{entre épithèse implanto-portée et reconstruction chirurgicale ou épithèse classique}

\section{Comparaison}

\section{avec la reconstruction chirurgicale}

Au plan chirurgical, le geste opératoire pour la pose des implants reste beaucoup plus simple, limité et bref. II peut aussi être effectué sous anesthésie locale $[2,36]$. Ce geste est donc beaucoup plus bénin pour le patient par comparaison avec une chirurgie reconstructrice. Ce traitement est aussi réversible, la dépose des piliers cutanés étant possible sans retirer les implants. Une reproduction fidèle de I'organe peut aussi être plus facilement obtenue par l'épithèse, surtout depuis I'avènement des silicones $[6,7]$.

Dans les processus carcinologiques, la prothèse implanto-portée permet de masquer très rapidement les zones d'exérèses, en comparaison des délais importants nécessaires aux reconstructions par lambeaux. Sur le plan économique, le coût global d'une reconstruction par épithèse implanto-portée est très inférieur. Les interventions sont réalisées en chirurgie ambulatoire, éventuellement sous anesthésie locale. S'y ajoute toutefois le coût des implants et de la prothèse, qui doit être renouvelée tous les deux ans en moyenne [15]. Les reconstructions chirurgicales restent des interventions lourdes avec en plus le risque lié à l'anesthésie générale. Le résultat final présente certes un aspect esthétique moins satisfaisant, mais évite l'impression d'inertie, de figé qui est ressentie parfois entre l'épithèse et les tissus vivants de voisinage [2].

\section{Comparaison}

\section{avec les épithèses}

classiques

Le confort et la sécurité du patient sont majorés par les épithèses implanto-portées par comparaison avec les épithèses classiques [37]. Celles-ci présentent en effet une stabilité et une rétention très améliorées avec une facilité de mise en place $[38,39]$. Les inconvénients des autres moyens de fixation tels que les adhésifs et les montures de lunette sont aussi éliminés $[12,40]$. Le camouflage de la perte de substance reste permanent, quels que soient les positions ou les mouvements de la tête, la présence de sueur ou de sécrétions (celles-ci pouvant décoller une prothèse retenue par de l'adhésif). Cette épithèse se détériore aussi moins rapidement ce qui justifie un renouvellement moins fréquent et donc un moindre coût. L'hygiène péri-implantaire tout comme le suivi doivent être rigoureux, ce qui nécessite une sélection des patients afin de ne pas compromettre le résultat ainsi obtenu [6]. 


\section{Conclusion}

La prothèse maxillo-faciale (PMF) permet de rétablir la morphologie du visage. L'objectif esthétique est primordial en masquant les mutilations. Les résultats cosmétiques des épithèses faciales ainsi que leur rétention et leur stabilité sont nettement améliorés par I'utilisation des implants. L'ancrage endo-osseux procure un confort et une sécurité au patient ce qui peut améliorer sa vie au quotidien, voire même lui permettre de s'adonner à une pratique sportive. La prothèse maxillo-faciale implanto-portée reste une alternative fiable et de bonne qualité à la chirurgie reconstructrice et mérite de figurer en bonne place parmi les moyens thérapeutiques à la disposition des chirurgiens. Le surcoût des implants se justifie car il est largement compensé par la fiabilité de la technique, la qualité de la rétention de la prothèse, la brièveté des hospitalisations et la satisfaction des patients. Seule une collaboration étroite et pluridisciplinaire de tous les acteurs, dont l'épiprothésiste, permet d'obtenir un résultat satisfaisant.

\section{Bibliographie}

1. Benoist M, Quentin PY. Histoire de la prothèse maxillo-faciale. Actualités Odonto-Stomatol 1998;202:179-193.

2. Compère JF, Sabin $P$, Labbé D, Pacini R. Épithèses faciales implanto-portées. Encycl Med Chir (Elsevier, Paris) Stomato Odonto 1, 22-087-P-10, 1996, 7 p.

3. Schneider Udo F, Gellich Nils-Claudiu S. Une épithèse ancrée sur des implants et attachements magnétiques. La réhabilitation de la région orbito-zygomatique. Présentation d'un cas. Rev Mens Suisse Odontostomatol 2002;112(4):342-354.

4. Maurice $D$, André $O$, Ravallec X, Lerouxel E, Yigitoglu M, Augier MC. Odontologie et prise en charge des défauts maxillo-faciaux. Les Cahiers de Prothèse déc 2002;120:17-25.

5. Sabin $P$, Labbé $D$, Compère JF. Épithèses maxillo-faciales sur implants endo-osseux. Différents modes de fixation. Rev Stomatol Chir Maxillofac 1993;94:82-86.

6. Pomar $\mathrm{P}$, Soulet $\mathrm{H}$. Mise en œuvre d'un élastomère de silicone dans la réalisation d'organes artificiels faciaux. Actualités Odonto-Stomatol 1996;193:79-91.

7. Vigarios $E$, Pomar $P$, Toulouse E, Fusaro $S$, Grhenassia C. Épithèses faciales. Encycl Méd-Chir (Elsevier SAS, Paris), Stomato 22-066-B-56, 2006, 10 p.

8. Tinel $P$, Pereyrol $C L$, Touitou M. Les adhésifs et les colles comme moyens de rétention des prothèses faciales externes.

Revue Française de Prothèse Maxillo-Faciale 1972;1:7-13.

9. Louis R, Marion DMD MS, Sandra L. Rothenberger, Glenn E. Minsley, DMD. A method of fabrication of a facial prosthesis that improves retention and durability: A clinical report. J Prosthet Dent 1997;77:457-460.

10. Pasturel A, Savy P, Pons J. La fixation par colle des prothèses faciales. Revue française de Prothèse Maxillo-Faciale 1972;15-20.

11. Bränemark PI, Zarb G, Albrekstsson T. Prothèses ostéo-intégrées. L'ostéo-intégration en pratique clinique. Paris : Éditions CdP 1998:352.

12. Karayazgan B, Gunay $Y$, Atay A, Noyun F. Facial defects restored with 
extraoral implant-supported protheses.

J Craniofac Surg

Sep;18(5):1086-1090.

13. Labbé D. et al.

Épithèses orbito-palpébrales et implants intra-osseux. Rev Stomatol Chir Maxillofac 1992;1:51-53.

14. Davarpanah $M$, Martinez $H$, Kebir M, Tecucianu JF. Manuel d'implantologie clinique.

Paris : Éditions CdP 1999:338.

15. Labbé $D$, Bénateau $H$, Compère JF, Sabin $P$. Implants extra-oraux: indications et contre-indications. Rev Stomatol Chir Maxillofac 2001;102(5):239-242.

16. Berdugo $\mathrm{H}$.

L'efficacité en implantologie. Paris : Éditions Masson 2007:162.

17. Bénateau $H$, Crasson $F$, Labbé D, Ricala S, Alix T. Implants extra-oraux et irradiation : tendances actuelles. Rev Stomatol Chir Maxillofac 2001;102(5):266-269.

18. Leonardi A, Buonaccorsi $S$, Pellacchia V, Moricca LM, Indrizzi E, Fini G. Maxillofacial prosthetic rehabilitation using extraoral implants. J Craniofac Surg 2008;19(2):398-405.

19. Jeffrey E Rubestein, DMD, MS.

Attachments used for implant-supported facial prostheses: A survey of United States, Canadian, and Swedish centers. J Prosthet Dent 1995;73(3):262-266.

20. Badie-Bodiri B, Kaplanski P. Implants extra-oraux : principales aires d'implantation.
Rev Stomatol Chir

Maxillofac

2001;102(5):229-233.

21. Schoen PJ, Raghoebar G, Reintsema $\mathrm{H}$, Burlage FR, Roodenburg JL, Vissink A. Treatment Outcome of Bone-Anchored Craniofacial Prostheses after Tumor Surgery. Cancer 2001;92(12):3045-3050.

22. Nishimura RD, Roumanas $E$, Moy PK, Sugai T, Earl G. Freymiller.

Osseointegrated implants and orbital defects:

U.C.L.A. experience.

J Prosthet Dent 1998;79(3):304-309.

23. Boudard $P$, Cadre B, Herman D, Krastinova D, Sabin P.

Pertes de substance

localisées.

Critères de recours aux implants extra-oraux et cas cliniques.

Rev Stomatol Chir

Maxillofac

2001;102(5):253-260.

24. Tjellström A, Portmann D. Application des implants ostéo-intégrés dans les épithèses et la prothèse auditive. Rev Laryngol 1992;113(5):439-445.

25. Fouassadier F, Menard R, Pillet J, Bertrand JC. Prothèse auriculaire fixée sur implants intra-mastoïdiens. À propos de 11 observations. Rev Stomatol Chir Maxillofac 1993;5:312-317.

26. Gary JJ, Donovan M. Retention designs for bone-anchored facial prostheses.

J Prosthet Dent 1993;70(4):329-332.

27. Wolfaardt J., Coss $P$, Levesque $\mathrm{R}$. Craniofacial osseointegration:
Technique for bar and acrylic resin substructure construction for auricular prostheses. J Prosthet Dent 1996;76(6):603-607.

28. Nishimura RD, Roumanas $E_{\star}$ Moy PK, Sugai T.

Nasal defects and osseointegrated implants: UCLA experience. J Prosthet Dent 1996;76(6):597-602.

29. Jensen OT, Brownd C, Blacker J.

Nasofacial prostheses supported by osseointegrated implants. Inter J Oral Maxillofac implants 1992;7(2):203-210.

30. Sabin P, Cadre B, Ferrand JF, Pacini R, Labbe D.

Les épithèses implanto-portées : technique et intérêt comparés des implants endo- et juxta-osseux. Rev Laryngol Oto Rhinol 1997;118(2):103-107.

31. Desmet $B$, Bonin B, Palmer S, Belloni D. Description et classification des implants extra-oraux. Rev Stomatol Chir Maxillofac 2001; 102(5):235-238.

32. Sabin $P$, Labbé $D$, Ferrand $J Y$, Kaluzinski E, Compère JF. Épithèses implanto-portées. Intérêts des implants endoet juxta-osseux. Rev Stomatol Chir Maxillofac 1997;4:248-252.

33. Brix M, Badie-Bodiri B, Delcampe $P$. Implants extra-oraux : procédures chirurgicales. Rev Stomatol Chir Maxillofac 2001;102(5):243-247.

34. Beumer J III, Lewis SG. La prothèse sur implants de Bränemark. Protocole clinique 
et technique de laboratoire. Paris, Éditions CDP 1991:250.

35. Pacini R, Poulain G, Bujeaud C, Benateau $\mathrm{H}$, Durand Ma, Durand Mi. Épithèses implanto-portées : intérêt de la collaboration chirurgien-épithésiste. Rev Stomatol Chir Maxillofac 2001; 102(5):270-273.

36. Disant $F$.

Place de l'épithèse dans les réhabilitations des pertes de substances de la face.

Rev Laryngol Oto Rhinol 1997;118(2):109-112.
37. Lemon JC, Chambers MS. Locking retentive attachment for an implant-retained auricular prosthesis. J Prosthet Dent 2002;87(3):336-338.

38. Chang TL, Neal G, Roumanas $\mathrm{E}$, Beumer J III, and LS. Treatment satisfaction with facial prostheses. J Prosthet Dent 2005;94(3):275-280.

39. Wright RF, Zemnick $C$, Wazen JJ, Asher E. Osseointegrated implants and auricular defects: a case series study. $\mathrm{J}$ Prosthodont 2008;17(6):468-475. Epub 2008 Jun 9.

40. Toljanic J, Eckert S, Roumanas $\mathrm{E}$, Beumer III J. Osseointegrated craniofacial implant in the rehabilitation of orbital defects: an update of a retrospective experience in the United States. J Prosthet Dent 94:177-182.

\section{SUMMARY}

\section{Implantology and facial epithesis}

Yomin Cécile ALLOH AMICHIA, François BRÉTÉCHÉ, Joël LANHOUET, Laurent LE GUEHENNEC, Pierre LE BARS

$$
\begin{aligned}
& \text { Keywords } \\
& \text { - maxillofacial prosthesis } \\
& \text { - implantology } \\
& \text { - extra-oral implants } \\
& \text { - implant-retained } \\
& \text { facial prosthesis }
\end{aligned}
$$

Facial epithesis still remain useful in several clinical situations. Classical «fixations" such as sealent, glue, glasses are now replaced by extra-oral bone anchored titan implants. Those are now a reliable and safe alternative offering better epithesis retention for facial maxilloprothesis. Prothesis can be fixed either by mechanical, magnetic or both fixations.

\section{Questions - Réponses}

\section{Implantologie et épithèses}

\section{Les épithèses implanto-portées sont indiquées :}

1 - en tant que solution d'attente à une reconstruction chirurgicale

2 - en alternative à toute reconstruction chirurgicale

3 - dans les cas d'échecs de reconstruction chirurgicale
La mise en place des implants pour épithèses :

4 - peut se faire sous anesthésie locale

5 - peut s'effectuer chez un patient quelque soit son état général

6 - est contre-indiquée dans un os fragilisé

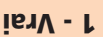

